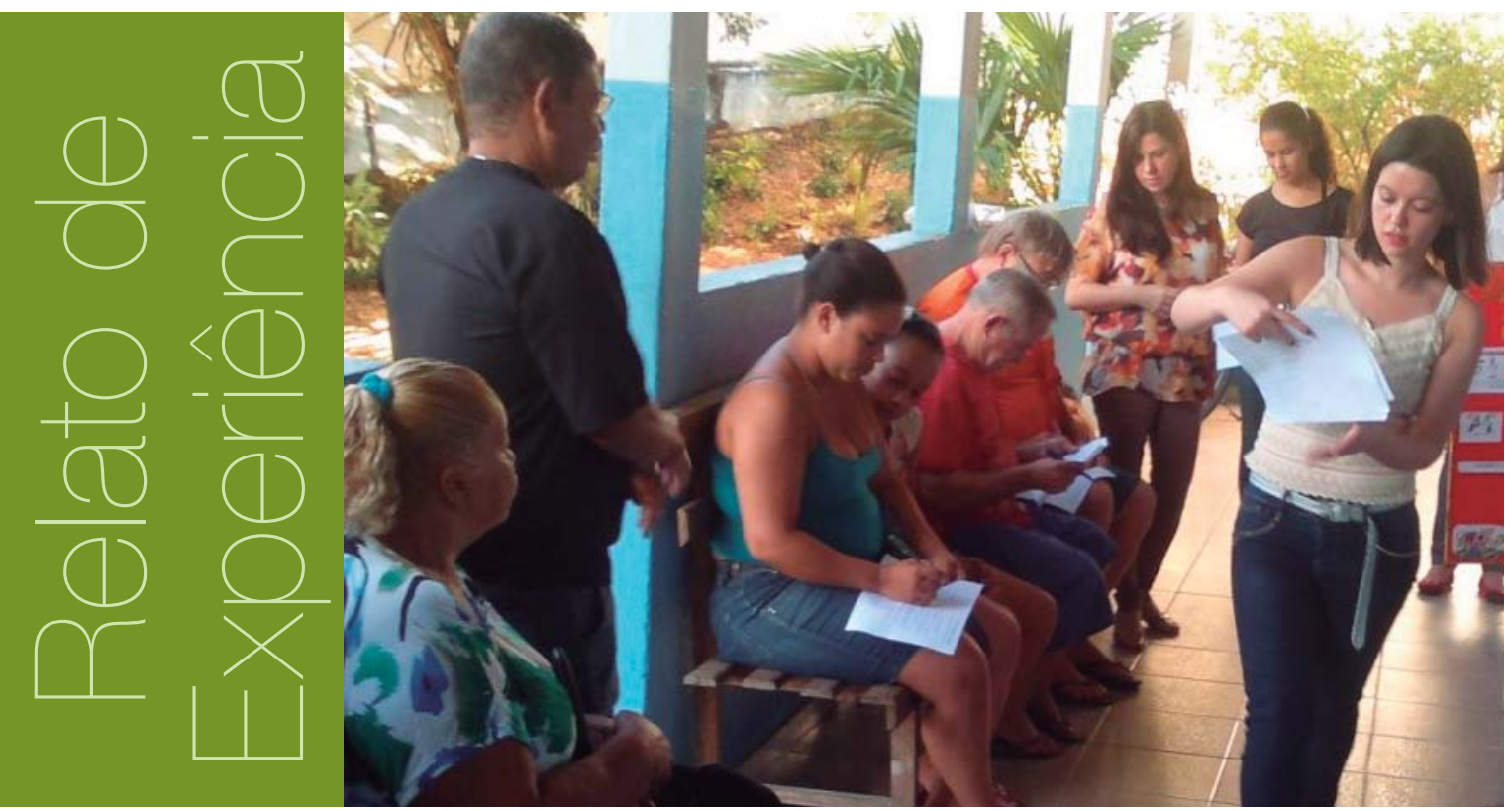

\title{
Projetos interdisciplinares
} de extensão universitária: possibilidades formativas no campo da saúde

Maria Gabriela Parenti Bicalho1 - maria.gabriela@ufjf.edu.br Bárbara de Cássia Vilela² - barbaradcassia@yahoo.com.br Luanna Miranda ${ }^{3}$ - luannamiranda11@yahoo.com.br Luiz Eduardo de Almeida4 - luiz.almeida@ufjf.edu.br

\section{RESUMO}

As demandas para a formação dos profissionais na área da saúde relacionam-se a um conceito ampliado de saúde. 0 Projeto "Equipe Sala de Espera: trabalho multiprofissional em Unidades de Atenção Primária à Saúde de Governador Valadares" é composto pelos cinco cursos da área da saúde do câmpus avançado da Universidade Federal de Juiz de Fora (UFJF) no referido município. São relatadas as atividades de educação em saúde no contexto de campanhas "Outubro Rosa" e "Novembro Azul".

\section{PALAVRAS-CHAVE}

Formação profissional. Extensão Universitária. Integração ensino-serviço. Saúde do homem. Saúde da mulher.

\footnotetext{
1 Doutora em Educação - Professora do Departamento de Medicina da Universidade Federal de Juiz de Fora - Câmpus Governador Valadares, MG

2 Acadêmica do curso de Medicina - Universidade Federal de Juiz de Fora, Câmpus Governador Valadares, MG

3 Acadêmica do curso de Fisioterapia - Universidade Federal de Juiz de Fora, Câmpus Governador Valadares, MG

4 Mestre em Clínica Odontológica - Professor do Departamento de Odontologia da Universidade Federal de Juiz de Fora - Câmpus Avançado de Governador Valadares
} 


\section{ABSTRACT}

The demands for the training of professionals in health are related to a broader concept of health. The project "Equipe Sala de Espera: trabalhomultiprofissionalemUnidades de AtençãoPrimária à Saúde de Governador Valadares " consists of five courses in the health field of advanced campus of the Universidade Federal de Juiz de Fora (UFJF) in that municipality. Health education activities are reported in the context of campaigns of "Outubro Rosa" e "Novembro Azul".

\section{KEYWORDS}

Professional training. University Extension. Integrating teaching and service .Men's health Women'shealth.

\section{Relato de experiência}

As transformações no conceito de saúde marcaram as discussões da área ao longo do século XX. Avançando em relação às concepções de saúde como ausência de doença, a Primeira Conferência Internacional sobre Promoção da Saúde, realizada em novembro de 1986 em Otawa, indica um conceito amplo de saúde. No Brasil, a discussão do conceito de saúde faz parte do arcabouço teórico que fundamenta as políticas públicas implementadas pelo Sistema Único de Saúde (SUS). Esse contexto apresenta novas demandas para a formação dos profissionais de saúde, voltadas para a atuação multi e interprofissional e a compreensão dos determinantes sociais dessa área. Os cursos de graduação na área da saúde procuram responder a essas novas demandas para a formação profissional, reelaborando seus projetos pedagógicos e buscando a inserção permanente dos estudantes nos cenários da prática.

0 atendimento a esses desafios é um dos objetivos do Projeto de Extensão "Equipe Sala de Espera: trabalho multiprofissional em Unidades de Atenção Primária à Saúde (UAPS) de Governador Valadares", realizado conjuntamente pelos cursos da área da saúde do câmpus avançado da Universidade Federal de Juiz de Fora (UFJF) no município de Governador Valadares, implantados a partir de 2012: Farmácia, Fisioterapia, Medicina, Nutrição e Odontologia. Professores e estudantes envolvidos realizam atividades formativas que possibilitem a convergência de conceitos e metodologias dos diferentes cursos, em parceria com a rede municipal de saúde. 0 desenvolvimento do projeto, idealizado e implantado a partir de 2012, levou ao estreitamento da comunicação ensino-serviço, culminando na inserção, em 2014, na agenda da Secretaria Municipal de Saúde, com a participação nas campanhas Outubro Rosa e Novembro Azul no ano de 2014, trazendo como recorte temporal o período de setembro a dezembro de 2014.

A Política Nacional de Atenção Integral à Saúde da Mulher, fundamentada inicialmente na atenção à gestação e ao puerpério, baseia-se, atualmente, na educação em saúde, prevenção, diagnóstico precoce e tratamento de patologias que as acometem (BRASIL, 2004). Em contrapartida, a menor expectativa de vida masculina evidencia a necessidade de maior atenção da saúde pública em relação aos homens. Essa atenção deve visar a aproximação do homem com 0 atendimento integrado em saúde, deixando de tratá-lo apenas por demandas espontâneas que envolvam doenças sexualmente transmissíveis, violência, condições severas e crônicas (VIEIRA et al., 2011), mas também trabalhar com a prevenção e tratamento. A Política Nacional de Atenção Integral à Saúde do Homem visa fundamentar ações em saúde voltadas para essas demandas. (BRASIL, 2008).

Diante de fatos explíitos da necessidade de trabalhos direcionados, pontualmente, para a saúde da mulher e do homem, foram instituídas campanhas, o Outubro Rosa e o Novembro Azul, desenvolvidos pela rede pública de saúde em consonância com o Ministério da Saúde e outras instituições públicas e privadas.

A partir do contato com a equipe de saúde da Estratégia de Saúde da Família e da definição da participação da equipe do Projeto - dois (duas) estudantes e um(a) professor(a) de cada um dos cinco cursos participantes - nas campanhas Outubro Rosa e Novembro Azul, as ações foram realizadas em três fases: planejamento, execução e avaliação. No planejamento, foi realizada capacitação da equipe em relação aos temas saúde da mulher e saúde do homem, por meio 
de leituras e discussões. A seguir, foram planejadas as ações de educação em saúde a serem desenvolvidas na Estratégia de Saúde da Família. Na fase de execução, as atividades foram desenvolvidas na UAPS do bairro Esperança (ESF-Esperança, ESF-Nossa Senhora das Graças e ESF-Distrito Sanitário III), no município de Governador Valadares, MG, abrangendo metodologias de abordagem dos usuários na sala de espera, estratégias de mobilização em relação às temáticas e de apresentação das informações. Foram adotadas estratégias metodológicas voltadas para a participação dos usuários e profissionais de saúde presentes, por meio da expressão de suas ideias e dúvidas acerca do tema. Na fase de avaliação, discutiu-se em reuniões 0 desenvolvimento das diversas atividades realizadas. 0 relato dos professores e estudantes foi analisado considerando-se como critérios de avaliação a capacidade de mobilização dos usuários presentes, a pertinência dos conteúdos apresentados e a participação dos usuários e profissionais, no momento da discussão. Após cada ação são afixadas na unidade, em um espaço específico, informações sobre o assunto discutido.

Figura 1: Equipe extensionista e equipe de saúde

Fonte: Projeto Sala de Espera: trabalho multiprofissional em Unidades de Atenção Primária à Saúde (UAPS) de Governador Valadares.

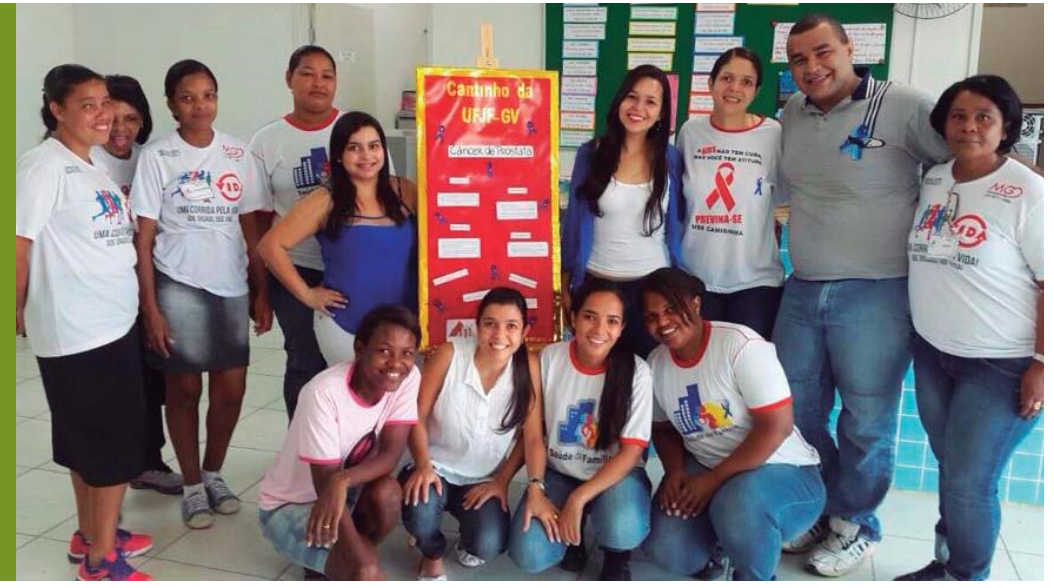

Figura 2: Painel montado na dinâmica realizada

Fonte: Projeto Sala de Espera: trabalho multiprofissional em Unidades de Atenção Primária à Saúde (UAPS) de Governador Valadares.

Figura 3: Realização de atividade de educação em saúde

Fonte: Projeto Sala de Espera: trabalho multiprofissional em Unidades de Atenção Primária à Saúde (UAPS) de Governador Valadares.
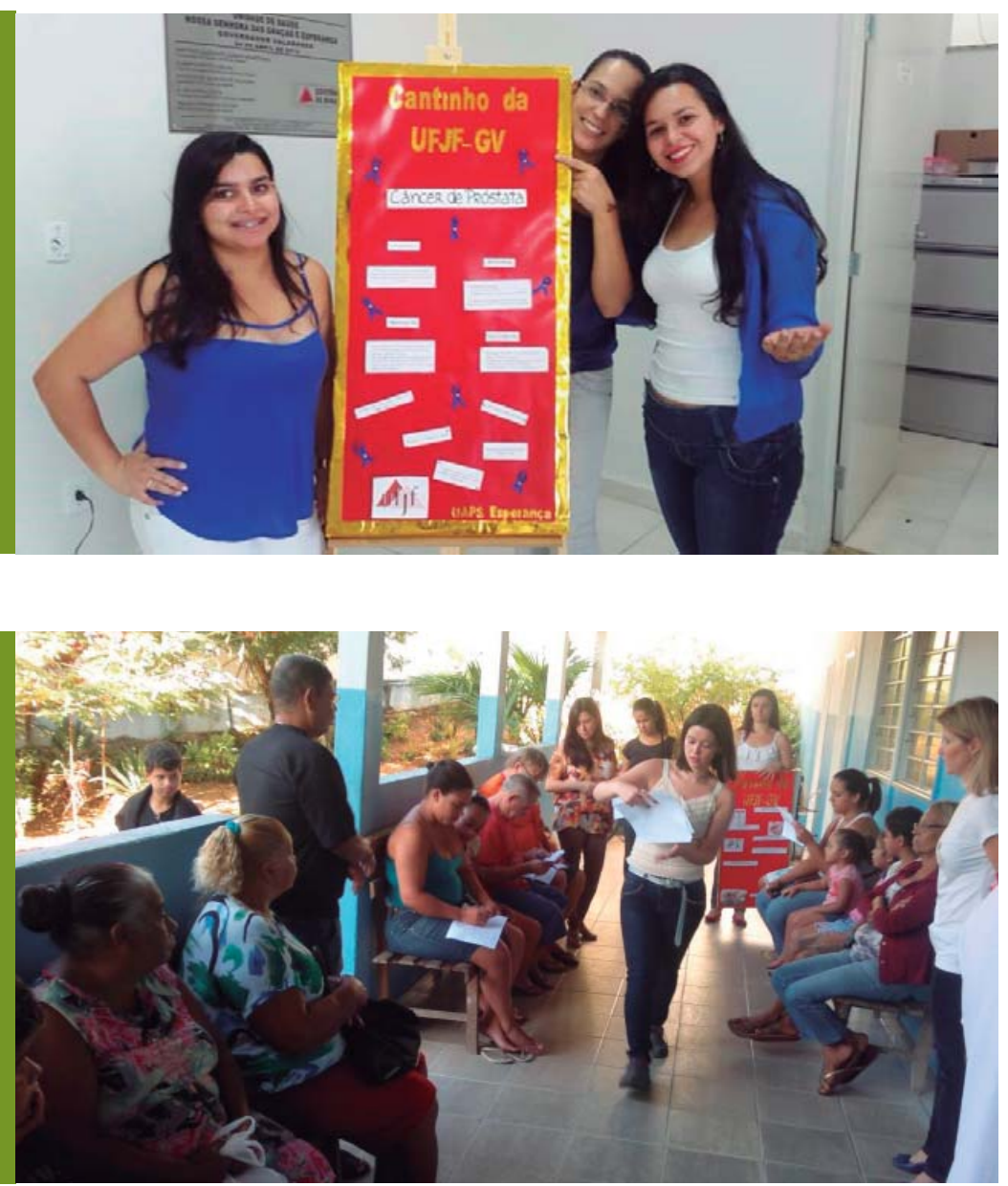
Nos meses outubro e novembro 2014, foram realizadas 10 (dez) ações de Educação em Saúde pela equipe do Projeto de Extensão Sala de Espera, da UFJF Câmpus Governador Valadares. A inserção das atividades universitárias na agenda do serviço de saúde possibilitou o aprofundamento da relação entre os cinco cursos da área da saúde envolvidos e a rede pública de saúde do município. A realização das atividades de forma conjunta, envolvendo cursos diferentes, exigiu de professores e estudantes a exposição e a discussão de conhecimentos teóricos e práticos de cada curso envolvido, propiciando o desenvolvimento paulatino de conhecimentos que integram as diferentes áreas e permitem, em alguns momentos, ultrapassar as barreiras entre elas, construindo saberes integrados. Entende-se que esse fator pode contribuir para a formação de profissionais de saúde preparados para o trabalho e a pesquisa multidisciplinares (SILVA, 2011; PEDUZZl et al, 2013). 0 desenvolvimento de projeto de extensão em uma Estratégia de Saúde da Família possibilitou o contato mais estreito dos professores e estudantes com a equipe de saúde e com os usuários, e o conhecimento das dinâmicas e da realidade da Atenção Primária. Ao planejar e executar ações de educação nesse contexto, os estudantes da área da saúde preparam-se para a atuação profissional sensível às possibilidades e às dificuldades do trabalho na rede pública de saúde.

\section{Referências}

BRASIL. Ministério da Saúde. Secretaria de Atenção à Saúde. Departamento de Ações Programáticas Estratégicas. Política Nacional de Atenção Integral à Saúde da Mulher: princípios e diretrizes. Brasília, 2004.

BRASIL. Ministério Da Saúde. Secretaria De Atenção à Saúde. Departamento De Ações Programáticas Estratégicas. Política Nacional de Atenção Integralà Saúde do Homem: princípios e diretrizes. Brasília, 2008.

PEDUZZI, Marina et al . Educação interprofissional: formação de profissionais de saude para 0 trabalho em equipe com foco nos usuários. Rev. esc. enferm. USP, São Paulo, v. 47, n. 4, p. 977-983, ago. 2013. Disponível em <http://www.scielo.br/scielo.acesso em 01 maio 2015.

SILVA, Rinaldo Henrique Aguilar. Educação interprofissional na graduação em saúde: aspectos avaliativos da implantação na Faculdade de Medicina de Marília (Famema). Educ. rev., Curitiban. 39, p. 159-175, abr. 2011. Disponível em <http://www.scielo.br/scielo.php acessos em 02 maio 2015.

VIEIRA,Luana Silva et al. A política nacional de saúde do homem: uma reflexão sobre a questão de gênero. Enfermagem em foco, São Paulo, v. 2, n.4, p. 215-217, nov 2011. 